\title{
NUMERICAL STUDY OF NATURAL CONVECTION IN AN ENCLOSED SQUARE CAVITY USING CONSTRAINED INTERPOLATED PROFILE (CIP) METHOD
}

\author{
Asish Mitra \\ Reviewer: Heat and Mass Transfer, WSEAS \\ Associate Professor\& HOD of Basic Sciences \& Humanities Department, \\ College of Engineering \& Management, Kolaghat, East Midnapur, West Bengal -721171 \\ mitra_asish@yahoo.com
}

\begin{abstract}
In the present study, Constrained Interpolated Profile (CIP) method was used to simulate the natural convection heat transfer and fluid flow in an enclosed square cavity with differentially heated side walls. The fundamental idea of this method is to solve the advection phase equation with CIP method and the non-advection phase equation is calculated with finite difference method. CIPNSE is applied to predict the temperature and velocity profiles in a square cavity for various Rayleigh number: Ra=10 $0^{3}, 10^{4}$ and $10^{5}$. The streamline and isotherms obtained under these conditions were then compared with those published in literature and found a good agreement.
\end{abstract}

Keywords: Constrained Interpolated Profile (CIP), Finite Difference Method (FDM) and Lattice Boltzmann Method (LBM), Natural Convection, Square Cavity, Stream-Function Vorticity.

\section{LITERATURE SURVEY:}

The natural or free convection is the phenomenon of heat transfer between a surface and a fluid moving over it with the fluid motion caused entirely by the buoyancy forces that arise due to the density changes that result from the temperature variations in the flow. Since the early works by researchers ([1], [2], [3], [4]) a great deal of theoretical and experimental researches was dedicated to investigate this phenomenon. The fundamental interest comes from the concern to understand the heat transfer mechanism ([5], [6],[7]) and fluid flow behavior around the surfaces ([8],[9]). On the other hand, a similar interest was provoked by the wide range of engineering applications utilizing this type of phenomenon ([10], [11]). Among the problems related to natural convection, many researchers focused their investigation on the heat transfer and fluid flow behavior from a differentially heated side walls in a cavity ([12], [13], [14]). They frequently considered adiabatic boundary condition for the top and bottom walls. However, very few investigated the effect of perfectly conducting top and bottom walls although it plays important roles in real engineering applications ([15]).

In present study, numerical investigation of natural convection in a square cavity is carried out by considering perfectly conducting boundary condition for top and bottom walls. The left and right walls were maintained at hot and cold temperature respectively. The objective of this paper is to extend the formulation of Constrained Interpolated Profile (CIP) method for Navier-Stokes equations to predict temperature and velocity profiles in a differentially heated square enclosure.

\section{List of symbols}
$\mathrm{C}_{\mathrm{p}^{-}}$Specific heat
g - Gravitational acceleration
$\mathrm{H}$ - Length of cavity
$\mathrm{k}$ - Thermal conductivity
L - Length
$\mathrm{p}$ - Pressure
$\mathrm{t}$ - Time
$\mathrm{T}$ - Temperature
$\mathrm{T}_{\mathrm{h}}$ - Surface wall with hot temperature
$\mathrm{T}_{\mathrm{c}}$ - Surface wall with cold temperature
$\mathrm{u}$ - Velocity in $\mathrm{x}$ direction
$\mathrm{U}$ - Dimensionless velocity in $\mathrm{x}$ direction
$\mathrm{v}$ - Velocity in y direction
$\mathrm{V}$ - Dimensionless velocity in y direction
$\mathrm{x}$ - Axial distance
$\mathrm{X}$ - Dimensionless axial distance
y - Vertical distance
Y - Dimensionless vertical distance 


\section{Dimensionless Parameters}

AR - Aspect Ratio

Gr - Grashof Number

Pr - Prandtl Number

$\mathrm{Ra}$ - Rayleigh Number

\section{Greek Symbols}

$\rho$ - Density

$\beta$ - Volumetric thermal expansion

$\tau$ - Dimensionless time

$\theta$ - Dimensionless temperature

$\mu$ - Dynamic viscosity

$v$ - Kinematic shear viscosity

$\alpha$ - Thermal diffusivity

$\omega$ - Vorticity

$\Omega$ - Dimensionless vorticity

$\psi$ - Stream function

$\Psi$ - Dimensionless stream function

$\nabla$. Nabla operator

\section{Superscript}

n - Current value

$\mathrm{n}+1-$ Next step value

* - Non advection phase value

\section{Subscript}

$\mathrm{i}$ - $\mathrm{x}$ direction node

$\mathrm{j}$ - $\mathrm{y}$ direction node

max $i$ - $x$ direction maximum node

$\max \mathrm{j}-\mathrm{y}$ direction maximum node

\section{FORMULATION, GOVERNING EQUATIONS AND NUMERICAL METHODS}

The conservation equations for $2 \mathrm{D}$ incompressible square cavity flow in Cartesian form (Fig 1) are:

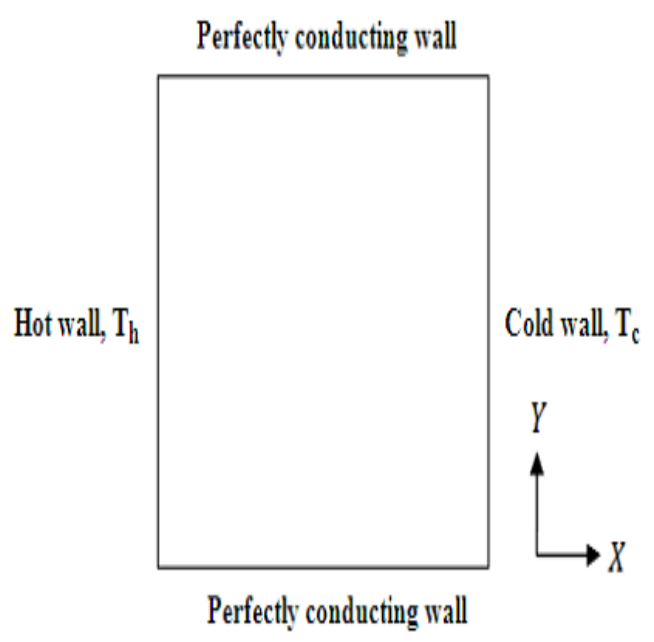

Fig1. Schematic geometry of a square cavity

$$
\frac{\partial u}{\partial x}+\frac{\partial v}{\partial y}=0
$$$$
\frac{\partial u}{\partial t}+u \frac{\partial u}{\partial x}+v \frac{\partial u}{\partial y}=-\frac{1}{\rho} \frac{\partial p}{\partial x}+v\left(\frac{\partial^{2} u}{\partial x^{2}}+\frac{\partial^{2} u}{\partial y^{2}}\right)
$$$$
2
$$

$$
\frac{\partial v}{\partial t}+u \frac{\partial v}{\partial x}+v \frac{\partial v}{\partial y}=-\frac{1}{\rho} \frac{\partial p}{\partial y}+v\left(\frac{\partial^{2} v}{\partial x^{2}}+\frac{\partial^{2} v}{\partial y^{2}}\right)+\beta g\left(T-T_{1}\right)
$$

3

$$
\frac{\partial T}{\partial t}+u \frac{\partial T}{\partial x}+v \frac{\partial T}{\partial y}=\frac{k}{\rho C_{p}}\left(\frac{\partial^{2} T}{\partial x^{2}}+\frac{\partial^{2} T}{\partial y^{2}}\right)
$$

The pressure terms are eliminated by taking the $y$ derivative of (3) and subtracting from it the $\mathrm{x}$-derivative of (2). This gives

$$
\begin{gathered}
\frac{\partial^{2} v}{\partial x \partial t}-\frac{\partial^{2} u}{\partial y \partial t}+u\left(\frac{\partial^{2} v}{\partial x^{2}}-\frac{\partial^{2} u}{\partial x \partial y}\right)+v\left(\frac{\partial^{2} v}{\partial y \partial x}-\frac{\partial^{2} u}{\partial y^{2}}\right)+\frac{\partial v}{\partial x}\left(\frac{\partial u}{\partial x}+\frac{\partial v}{\partial y}\right)-\frac{\partial u}{\partial y}\left(\frac{\partial u}{\partial x}+\frac{\partial v}{\partial y}\right) \\
=v\left[\left(\frac{\partial^{3} v}{\partial x^{3}}-\frac{\partial^{3} u}{\partial x^{2} \partial y}\right)+\left(\frac{\partial^{3} v}{\partial x \partial y^{2}}-\frac{\partial^{3} u}{\partial y^{3}}\right)\right]+\beta g \frac{\partial T}{\partial x} \\
5
\end{gathered}
$$

Using the definitions of stream function $(\psi)$ and vorticity $(\omega)$ 


$$
\begin{aligned}
& u=\frac{\partial \psi}{\partial y} \\
& v=-\frac{\partial \psi}{\partial x} \\
& \omega=\frac{\partial v}{\partial x}-\frac{\partial u}{\partial y}
\end{aligned}
$$

$$
\Omega=-\left[\frac{\partial^{2} \Psi}{\partial X^{2}}+\frac{\partial^{2} \Psi}{\partial Y^{2}}\right]
$$

$$
\frac{\partial \theta}{\partial \tau}+U \frac{\partial \theta}{\partial X}+V \frac{\partial \theta}{\partial Y}=\frac{\partial^{2} \theta}{\partial X^{2}}+\frac{\partial^{2} \theta}{\partial Y^{2}}
$$

Eq (5) can be written as

$$
\frac{\partial \omega}{\partial t}+u \frac{\partial \omega}{\partial x}+v \frac{\partial \omega}{\partial y}=v\left(\frac{\partial^{2} \omega}{\partial x^{2}}+\frac{\partial^{2} \omega}{\partial y^{2}}\right)+\beta g \frac{\partial T}{\partial x}
$$

9

In terms of the stream function, the equation defining the vorticity (8) becomes

$$
w=-\left(\frac{\partial^{2} \psi}{\partial x^{2}}+\frac{\partial^{2} \psi}{\partial y^{2}}\right)
$$

The following dimensionless variables are now introduced:

$$
\begin{aligned}
\Psi & =\frac{\psi P r}{v} \\
\Omega & =\frac{\omega H^{2} P r}{v} \\
X & =\frac{x}{H}, \quad Y=\frac{y}{H} \\
\tau & =\frac{t \alpha}{H} \quad U=\frac{u H}{\alpha}, \quad V=\frac{v}{\alpha} \\
\theta & =\frac{T-T_{C}}{T_{H}-T_{C}}
\end{aligned}
$$

Where

$$
\operatorname{Pr}=\frac{v}{\alpha}, \quad R a=\frac{\beta g\left(T_{H}-T_{c)} H^{3}\right.}{v \alpha}
$$

\section{CONSTRAINED INTERPOLATED PROFILE NAVIER STOKES EQUATION (CIPNSE)}

In the CIPNSE method, the equation is divided into two parts: advection phase and non advection phase. The non advection phase will be solved independently through finite difference method while the solutions of advection phase will be obtained using two-dimensional (2D) CIP method.

\section{Solution of Vorticity Transport Equation}

The vorticity transport equation (17) can be written as

$\frac{\partial \Omega}{\partial \tau}+U \frac{\partial \Omega}{\partial X}+V \frac{\partial \Omega}{\partial Y}=D$

Where

$D=\operatorname{Pr}\left[\frac{\partial^{2} \Omega}{\partial X^{2}}+\frac{\partial^{2} \Omega}{\partial Y^{2}}\right]+R a \cdot \operatorname{Pr}\left(\frac{\partial \theta}{\partial X}\right)$

The $\mathrm{X}$ - and Y-derivatives of (20) are

$$
\frac{\partial}{\partial X}\left[\frac{\partial \Omega}{\partial \tau}\right]+U \frac{\partial}{\partial X}\left[\frac{\partial \Omega}{\partial X}\right]+V \frac{\partial}{\partial X}\left[\frac{\partial \Omega}{\partial Y}\right]=\frac{\partial}{\partial X}[D]-\frac{\partial \Omega}{\partial X} \frac{\partial U}{\partial X}-\frac{\partial \Omega}{\partial Y} \frac{\partial V}{\partial X}
$$

$$
\frac{\partial}{\partial Y}\left[\frac{\partial \Omega}{\partial \tau}\right]+U \frac{\partial}{\partial Y}\left[\frac{\partial \Omega}{\partial X}\right]+V \frac{\partial}{\partial Y}\left[\frac{\partial \Omega}{\partial Y}\right]=\frac{\partial}{\partial Y}[D]-\frac{\partial \Omega}{\partial X} \frac{\partial U}{\partial Y}-\frac{\partial \Omega}{\partial Y} \frac{\partial V}{\partial Y}
$$

Putting the X-and Y-derivatives of D from (21) into (22) and (23) we get 
$\frac{\partial}{\partial X}\left(\frac{\partial \Omega}{\partial \tau}\right)+U \frac{\partial}{\partial X}\left(\frac{\partial \Omega}{\partial X}\right)+V \frac{\partial}{\partial X}\left(\frac{\partial \Omega}{\partial Y}\right)=\operatorname{Pr}\left(\frac{\partial^{3} \Omega}{\partial X^{3}}+\frac{\partial^{3} \Omega}{\partial X \partial Y^{2}}\right)+R a \cdot \operatorname{Pr}\left(\frac{\partial^{2} \theta}{\partial X^{2}}\right)-\frac{\partial \Omega}{\partial X} \frac{\partial U}{\partial X}-\frac{\partial \Omega}{\partial Y} \frac{\partial V}{\partial X}$
$\frac{\partial}{\partial Y}\left(\frac{\partial \Omega}{\partial \tau}\right)+U \frac{\partial}{\partial Y}\left(\frac{\partial \Omega}{\partial X}\right)+V \frac{\partial}{\partial Y}\left(\frac{\partial \Omega}{\partial Y}\right)=\operatorname{Pr}\left(\frac{\partial^{3} \Omega}{\partial X^{2} \partial Y}+\frac{\partial^{3} \Omega}{\partial Y^{3}}\right)+R a \cdot \operatorname{Pr}\left(\frac{\partial^{2} \theta}{\partial X}\right)-\frac{\partial \Omega}{\partial X} \frac{\partial U}{\partial Y}-\frac{\partial \Omega}{\partial Y} \frac{\partial V}{\partial Y}$

\section{Non-advection phase}

$\frac{\partial \Omega}{\partial \tau}=\operatorname{Pr}\left(\frac{\partial^{2} \Omega}{\partial X^{2}}+\frac{\partial^{2} \Omega}{\partial Y^{2}}\right)+R a \cdot \operatorname{Pr}\left(\frac{\partial \theta}{\partial X}\right)$

26

$\frac{\partial}{\partial X}\left(\frac{\partial \Omega}{\partial \tau}\right)=\operatorname{Pr}\left(\frac{\partial^{3} \Omega}{\partial X^{3}}+\frac{\partial^{3} \Omega}{\partial X \partial Y^{2}}\right)+R a \cdot \operatorname{Pr}\left(\frac{\partial^{2} \theta}{\partial X^{2}}\right)-\frac{\partial \Omega}{\partial X} \frac{\partial U}{\partial X}-\frac{\partial \Omega}{\partial Y} \frac{\partial V}{\partial X}$ 27

$\frac{\partial}{\partial Y}\left(\frac{\partial \Omega}{\partial \tau}\right)=\operatorname{Pr}\left(\frac{\partial^{3} \Omega}{\partial X^{2} \partial Y}+\frac{\partial^{3} \Omega}{\partial Y^{3}}\right)+R a \cdot \operatorname{Pr}\left(\frac{\partial^{2} \theta}{\partial X \partial Y}\right)-\frac{\partial \Omega}{\partial X} \frac{\partial U}{\partial Y}-\frac{\partial \Omega}{\partial Y} \frac{\partial V}{\partial Y}$ 28

Eqs (26) - (28) are solved by using central finite difference method (FDM), which finally gives

$$
\begin{aligned}
\Omega_{i, j}^{*}=\Omega_{i, j}^{n}+ & \Delta T \cdot \operatorname{Pr}\left(\frac{\Omega_{i+1, j}^{n}-2 \Omega_{i, j}^{n}+\Omega_{i-1, j}^{n}}{(\Delta X)^{2}}+\frac{\Omega_{i, j+1}^{n}-2 \Omega_{i, j}^{n}+\Omega_{i, j-1}^{n}}{(\Delta Y)^{2}}\right) \\
& +\Delta T \cdot \operatorname{Ra} \cdot \operatorname{Pr}\left(\frac{\theta_{i+1, j}^{n}-\theta_{i-1, j}^{n}}{2 \Delta X}\right)
\end{aligned}
$$

29

$$
\begin{aligned}
\frac{\partial}{\partial X}\left(\Omega_{i, j}^{*}\right)= & \frac{\partial}{\partial X}\left(\Omega_{i, j}^{n}\right) \\
& +\Delta T\left[\operatorname { P r } \left(\frac{\Omega_{i+2, j}^{n}-2 \Omega_{i+1, j}^{n}+2 \Omega_{i-1, j}^{n}-\Omega_{i-2, j}^{n}}{2 \Delta X^{3}}\right.\right. \\
& \left.+\frac{\Omega_{i+1, j+1}^{n}-2 \Omega_{i+1, j}^{n}+\Omega_{i+1, j-1}^{n}-\Omega_{i-1, j+1}^{n}+2 \Omega_{i-1, j}^{n}-\Omega_{i-1, j-1}^{n}}{2 \Delta X(\Delta Y)^{2}}\right) \\
& \left.+\operatorname{Ra} \cdot \operatorname{Pr}\left(\frac{\theta_{i+1, j}^{n}-2 \theta_{i, j}^{n}+\theta_{i-1, j}^{n}}{(\Delta X)^{2}}\right)\right] \\
& -\left(\frac{\Omega_{i+1, j}^{n}-\Omega_{i-1, j}^{n}}{2 \Delta X}\right)\left(\frac{U_{i+1, j}^{n}-U_{i-1, j}^{n}}{2 \Delta X}\right) \Delta T \\
& -\left(\frac{\Omega_{i, j+1}^{n}-\Omega_{i, j-1}^{n}}{2 \Delta Y}\right)\left(\frac{V_{i,+1 j}^{n}-V_{i-1, j}^{n}}{2 \Delta X}\right) \Delta T
\end{aligned}
$$

30

$$
\begin{aligned}
& \frac{\partial}{\partial Y}\left(\Omega_{i, j}^{*}\right) \\
& =\frac{\partial}{\partial Y}\left(\Omega_{i, j)}^{n}\right. \\
& +\Delta T\left[\operatorname{Pr}\left(\frac{\Omega_{i+1, j+1}^{n}-2 \Omega_{i, j+1}^{n}+\Omega_{i-1, j+1}^{n}-\Omega_{i+1, j-1}^{n}+2 \Omega_{i, j-1}^{n}-\Omega_{i-1, j-1}^{n}}{2 \Delta Y(\Delta X)^{2}}\right)\right. \\
& \left.+\frac{\Omega_{i, j+2}^{n}-2 \Omega_{i, j+1}^{n}+2 \Omega_{i, j-1}^{n}-\Omega_{i, j-2}^{n}}{2 \Delta Y^{3}}\right)
\end{aligned}
$$$$
\left.+\operatorname{Ra} \cdot \operatorname{Pr}\left(\frac{\theta_{i+1, j+1}^{n}-\theta_{i+1, j-1}^{n}-\theta_{i-1, j+1}^{n}+\theta_{i-1, j-1}^{n}}{4 \Delta X \Delta Y}\right)\right]
$$$$
-\left(\frac{\Omega_{i+1, j}^{n}-\Omega_{i-1, j}^{n}}{2 \Delta X}\right)\left(\frac{U_{i, j+1}^{n}-U_{i, j-1}^{n}}{2 \Delta Y}\right) \Delta T
$$$$
-\left(\frac{\Omega_{i, j+1}^{n}-\Omega_{i, j-1}^{n}}{2 \Delta Y}\right)\left(\frac{V_{i, j+1}^{n}-V_{i, j-1}^{n}}{2 \Delta Y}\right) \Delta T
$$$$
31
$$

\section{Advection phase}

$$
\begin{aligned}
& \frac{\partial \Omega}{\partial \tau}+U \frac{\partial \Omega}{\partial X}+V \frac{\partial \Omega}{\partial Y}=0 \\
& \frac{\partial}{\partial X}\left[\frac{\partial \Omega}{\partial \tau}\right]+\frac{\partial}{\partial X}\left[U \frac{\partial \Omega}{\partial X}\right]+\frac{\partial}{\partial X}\left[V \frac{\partial \Omega}{\partial Y}\right]=0 \\
& \frac{\partial}{\partial Y}\left[\frac{\partial \Omega}{\partial \tau}\right]+\frac{\partial}{\partial Y}\left[U \frac{\partial \Omega}{\partial X}\right]+\frac{\partial}{\partial Y}\left[V \frac{\partial \Omega}{\partial Y}\right]=0
\end{aligned}
$$

In CIP method, the profile between the lattice points is interpolated using cubic polynomial as in eq (35)

$$
\begin{aligned}
F_{i, j}(X, Y)= & {\left[\left(a_{1} \hat{X}+a_{2} \hat{Y}+a_{3}\right) \hat{Y}+a_{4} \hat{Y}+\Omega_{x}\right] \hat{X} } \\
& +\left[\left(a_{5} \hat{Y}+a_{6} \hat{X}+a_{7}\right) \hat{H}+\Omega_{y}\right]+\Omega \\
& 35
\end{aligned}
$$

Where

$$
\hat{X}=X-X_{i, j}, \text { and } \hat{Y}=Y-Y_{i, j}
$$


The coefficients of a1 ,a2,...a7 are determined so that the interpolation function and its first derivatives are continuous at both ends [16]. With this restriction, the numerical diffusion can be greatly reduced when the interpolated profile is constructed. The spatial derivatives are then calculated as

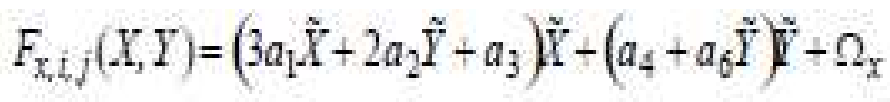

$$
F_{y, i, j}(X, Y)=\left(2 a_{2} \hat{Y}+a_{3}\right) \tilde{Y}+\left(3 a_{5} \tilde{Y}+2 a_{6} \tilde{X}+2 a_{7}\right) \tilde{Y}+\Omega_{y}
$$

38

In two-dimensional case, the adverted profile is approximated as follow

$$
\begin{aligned}
& \Omega_{L, j}^{n}=F_{i_{i}, j}(X+\eta Y+\xi) \\
& \Omega_{x, L j}^{n}=F_{x, L, j}(X+\eta Y+\xi) \\
& \Omega_{y, L, j}^{n}=F_{y, L, j}(X+\eta Y+\xi)
\end{aligned}
$$

Where

$$
\eta=-U_{\Delta} \tau \text { and } \xi=-V \Delta \tau \text {. }
$$

The newly calculated spatial quantities are then be used to solve non-advection phase of Eqns. (26) to (28) and vorticity formulation of Eqn. (18). In present study, the explicit central finite different discretisation method is applied with second order accuracy in time and space. For example, the treatment for eqn. (18) is

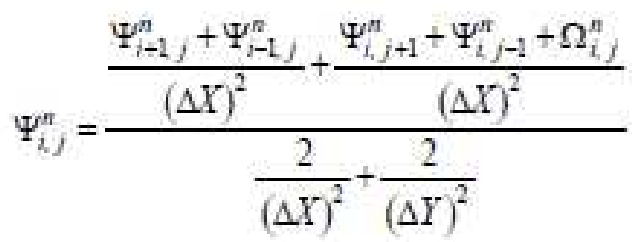

43

In summary, the evolution of the proposed scheme consists of three steps.

1. The initial value of $\Omega_{i, j}^{n}, \Omega_{x, i, j}^{n}$ and $\Omega_{y, i, j}^{n}$ are specified at each grid point.
2. Solve for $\Omega_{i, j}^{n^{*}} \Omega_{x, i, j}^{n^{*}}$ and $\Omega_{y, i, j}^{n^{*}}$ using the constrained interpolation process [from eqs (39), (40) and (41) ].

3. The values of $\Omega_{i, j}^{n+1}, \Omega_{x, i, j}^{n+1}$ and $\Omega_{y, i, j}^{n+1}$ are then computed from the newly advected values in step 2 by solving the nonadvection phase of the governing equations [from eqs (29) and (31) ].

Then the interpolation and the advection processes are repeated.

\section{Solution of Energy Equation}

The energy equation (19) can be written as

$$
\frac{\partial \theta}{\partial \tau}+U \frac{\partial \theta}{\partial X}+V \frac{\partial \theta}{\partial Y}=M
$$

Where

$$
M=\frac{\partial^{2} \theta}{\partial X^{2}}+\frac{\partial^{2} \theta}{\partial Y^{2}}
$$

The $\mathrm{X}$ - and Y-derivatives of (44) are

$$
\frac{\partial}{\partial X}\left[\frac{\partial \theta}{\partial \tau}\right]+U \frac{\partial}{\partial X}\left[\frac{\partial \theta}{\partial X^{2}}\right]+V \frac{\partial}{\partial X}\left[\frac{\partial \theta}{\partial X}\right]=\frac{\partial}{\partial X}\left[M-\frac{\partial \theta \partial}{\partial X} \frac{\partial}{\partial X} \frac{\partial \partial \partial}{\partial x} \frac{\partial}{\partial X}\right.
$$

$\frac{\partial}{\partial T}\left[\frac{\partial \theta}{\partial \tau}\right]+U \frac{\partial}{\partial X}\left[\frac{\partial \theta}{\partial X}\right]+V \frac{\partial}{\partial T}\left[\frac{\partial \theta}{\partial T}\right]=\frac{\partial}{\partial T}[M]-\frac{\partial \theta}{\partial X} \frac{\partial U}{\partial Y}-\frac{\partial \theta}{\partial V} \frac{\partial V}{\partial Y}$ 47

Putting the $\mathrm{X}$ - and Y-derivatives of M from (45) into (46) and (47) we get

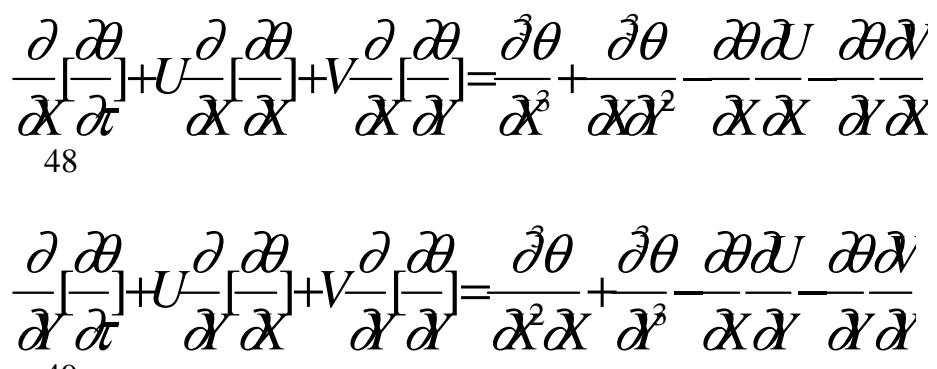

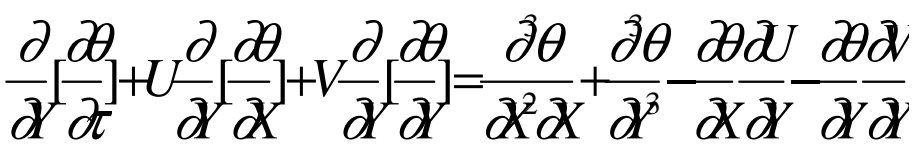




\section{Non-advection phase}

$\frac{\partial \theta}{\partial \tau}=\frac{\partial^{2} \theta}{\partial X^{2}}+\frac{\partial^{2} \theta}{\partial Y^{2}}$

50

$\frac{\partial}{\partial X}\left[\frac{\partial \theta}{\partial \tau}\right]=\frac{\partial^{3} \theta}{\partial X^{3}}+\frac{\partial^{3} \theta}{\partial X \partial Y^{2}}-\frac{\partial \theta}{\partial X} \frac{\partial U}{\partial X}-\frac{\partial \theta}{\partial Y} \frac{\partial V}{\partial X}$

$\frac{\partial}{\partial Y}\left[\frac{\partial \theta}{\partial \tau}\right]=\frac{\partial^{3} \theta}{\partial X^{2} \partial X}+\frac{\partial^{3} \theta}{\partial Y^{3}}-\frac{\partial \theta}{\partial X} \frac{\partial U}{\partial Y}-\frac{\partial \theta}{\partial Y} \frac{\partial V}{\partial Y}$

52

Eqs (50) - (52) are solved by using central finite difference method (FDM), which finally gives

$$
\theta_{i, j}^{*}=\theta_{i, j}^{n}+\Delta T\left(\frac{\theta_{i+1, j}^{n}-2 \theta_{i, j}^{n}+\theta_{i-1, j}^{n}}{(\Delta X)^{2}}+\frac{\theta_{i, j+1}^{n}-2 \theta_{i, j}^{n}+\theta_{i, j-1}^{n}}{(\Delta Y)^{2}}\right)
$$

$$
\begin{aligned}
\frac{\partial}{\partial X}\left(\theta_{i, j}^{*}\right)=\frac{\partial}{\partial X}\left(\theta_{i, j}^{n}\right) & \\
+ & \Delta T\left[\frac{\theta_{i+2, j}^{n}-2 \theta_{i+1, j}^{n}+2 \theta_{i-1, j}^{n}-\theta_{i-2, j}^{n}}{2 \Delta X^{3}}\right. \\
& \left.+\frac{\theta_{i+1, j+1}^{n}-2 \theta_{i+1, j}^{n}+\theta_{i+1, j-1}^{n}-\theta_{i-1, j+1}^{n}+2 \theta_{i-1, j}^{n}-\theta_{i-1, j-1}^{n}}{2 \Delta X(\Delta Y)^{2}}\right] \\
& -\left(\frac{\theta_{i+1, j}^{n}-\theta_{i-1, j}^{n}}{2 \Delta X}\right)\left(\frac{U_{i+1, j}^{n}-U_{i-1, j}^{n}}{2 \Delta X}\right) \Delta T \\
& -\left(\frac{\theta_{i, j+1}^{n}-\theta_{i, j-1}^{n}}{2 \Delta Y}\right)\left(\frac{V_{i,+1 j}^{n}-V_{i-1, j}^{n}}{2 \Delta X}\right) \Delta T
\end{aligned}
$$

$\frac{\partial}{\partial Y}\left(\theta_{i, j}^{*}\right)=\frac{\partial}{\partial Y}\left(\theta_{i, j}^{n}\right)$

$$
\begin{aligned}
& +\Delta T\left[\frac{\theta_{i+1, j+1}^{n}-2 \theta_{i, j+1}^{n}+\theta_{i-1, j+1}^{n}-\theta_{i+1, j-1}^{n}+2 \theta_{i, j-1}^{n}-\theta_{i-1, j-1}^{n}}{2 \Delta Y(\Delta X)^{2}}\right. \\
& \left.+\frac{\theta_{i, j+2}^{n}-2 \theta_{i, j+1}^{n}+2 \theta_{i, j-1}^{n}-\theta_{i, j-2}^{n}}{2 \Delta Y^{3}}\right] \\
& -\left(\frac{\theta_{i+1, j}^{n}-\theta_{i-1, j}^{n}}{2 \Delta X}\right)\left(\frac{U_{i, j+1}^{n}-U_{i, j-1}^{n}}{2 \Delta Y}\right) \Delta T
\end{aligned}
$$

$$
-\left(\frac{\theta_{i, j+1}^{n}-\theta_{i, j-1}^{n}}{2 \Delta Y}\right)\left(\frac{V_{i, j+1}^{n}-V_{i, j-1}^{n}}{2 \Delta Y}\right) \Delta T
$$

\section{Advection phase}

$$
\frac{\partial \theta}{\partial \tau}+U \frac{\partial \theta}{\partial X}+V \frac{\partial \theta}{\partial Y}=0
$$

$$
\frac{\partial}{\partial X}\left[\frac{\partial \theta}{\partial \tau}\right]+\frac{\partial}{\partial X}\left[U \frac{\partial \theta}{\partial X}\right]+\frac{\partial}{\partial X}\left[V \frac{\partial \theta}{\partial Y}\right]=0
$$

$$
\frac{\partial}{\partial Y}\left[\frac{\partial \theta}{\partial \tau}\right]+\frac{\partial}{\partial Y}\left[U \frac{\partial \theta}{\partial X}\right]+\frac{\partial}{\partial Y}\left[V \frac{\partial \theta}{\partial Y}\right]=0
$$

58

The same procedure is applied for getting the advection phase of energy equation.

\section{RESULTS AND DISCUSSION}

In this numerical research work, the natural convection phenomena in an enclosed square cavity have been studies using CIP method. Applying the numerical procedure mentioned in the last section, the streamlines and isotherms plots are obtained for various values for Rayleigh Numbers of $\mathrm{Ra}=103,104$ and 105 at a fixed Prandtl Number of 0.71. An in-house code (in Matlab) has been developed for the whole simulation. These are compared with those obtained from Lattice Boltzmann method (LBM) [17]. Figure 2 illustrates the comparison of the streamline plots for various Rayleigh number between the present and LBM methods, while Figure 3 demonstrates the comparison of the isotherm plots.

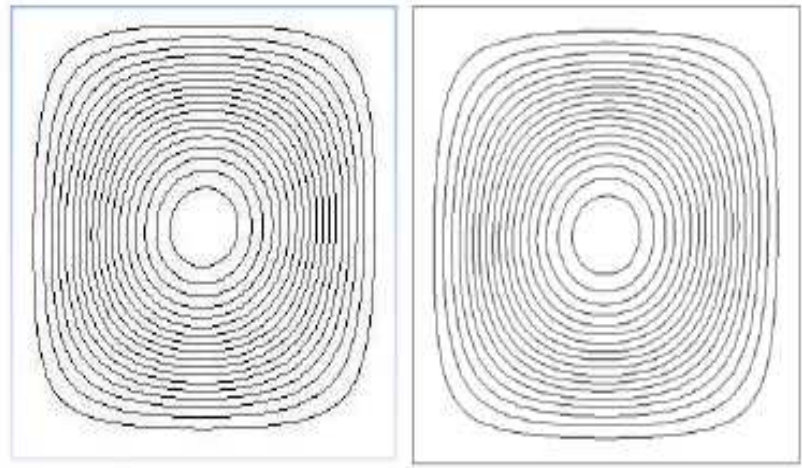

$\mathrm{Ra}=10^{3}$ 


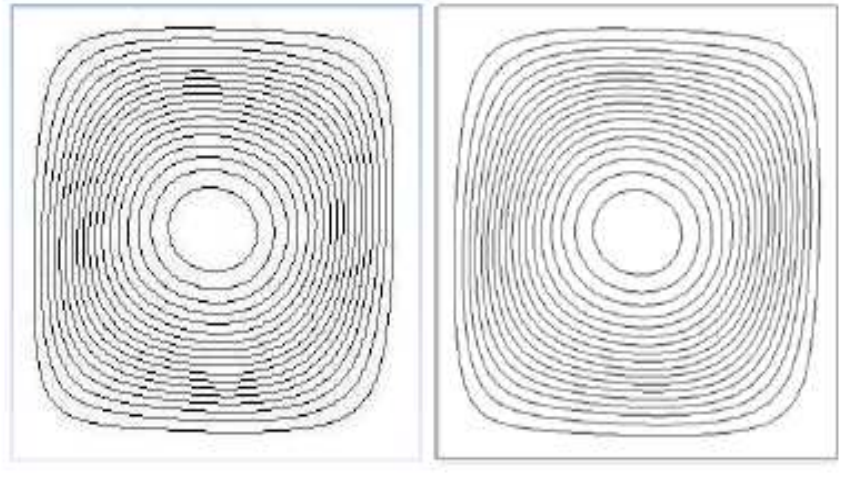

$\mathrm{Ra}=10^{4}$

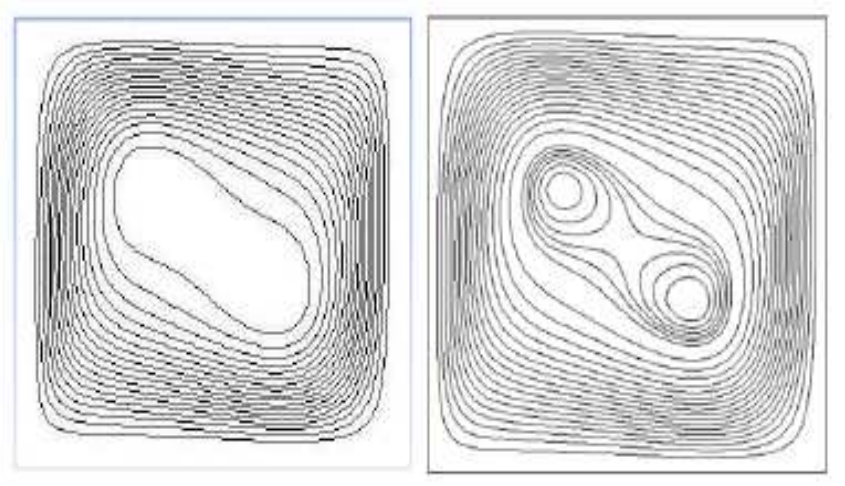

$$
\mathrm{Ra}=10^{5}
$$

Fig 2 Comparison of the streamline plots for various number between the present CIP (left) and LBM (right)

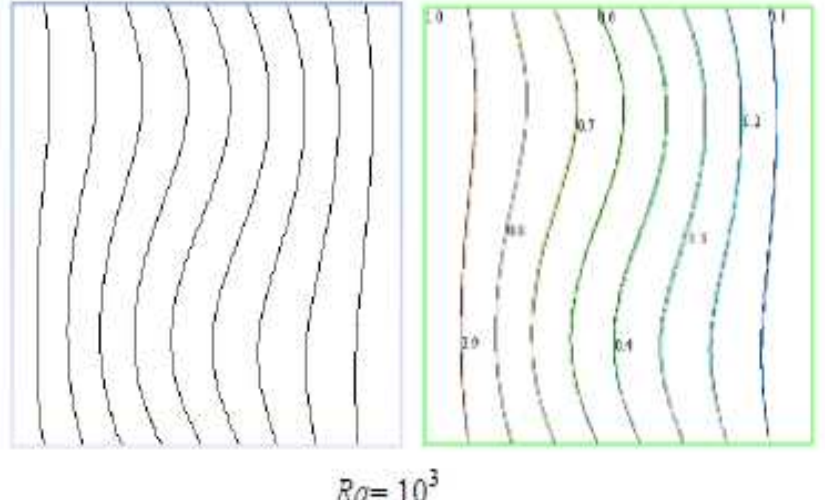

$R a=10^{3}$

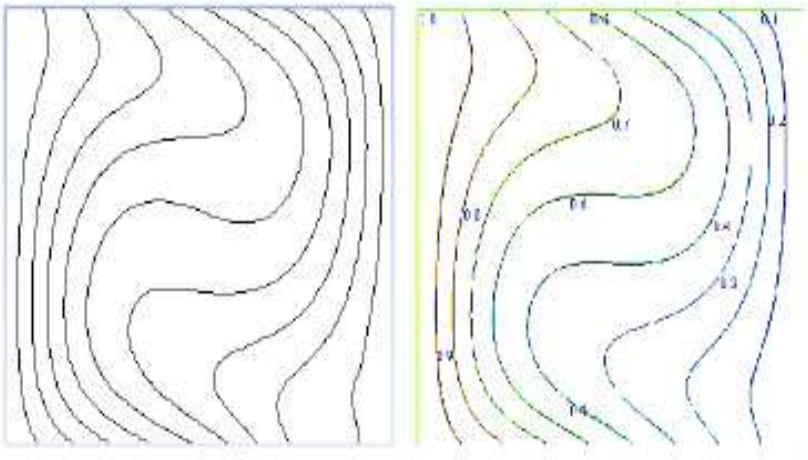

$R a=10^{4}$

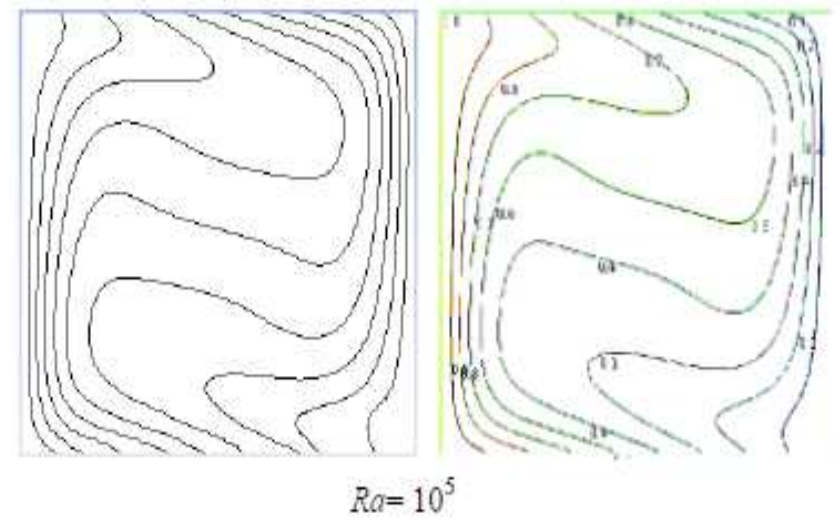

Fig 2 Comparison of the isotherm plots for various number between the present CIP (left) and LBM (right)

\section{CONCLUSIONS}

Natural convection in a square cavity was studied using CIPNSE method. The velocity and temperature profiles for Rayleigh numbers 103, 104 and 105 obtained in this approach were compared with those obtained from lattice-Boltzmann formulations. A good agreement between the present results and the past indicates that like finite difference and latticeBoltzmann formulations, the CIPNSE method may be an efficient and stable numerical scheme in natural convection. 


\section{REFERENCES}

[1] Lee, S.L., Hellman, J.M., 1969. Study of Firebrand Trajectories in a Turbulent Swirling Natural Convection Plume, Combustion and Flame 13, 645-655.

[2] Clifton, J.V., Chapman, A.J., 1969. Natural Convection on a Finite-Size Horizontal Plate, International Journal of Heat and Mass Transfer 12, 1573-1584.

[3] Hassan, K.E., Mohamed, S.A., 1970. Natural Convection from Isothermal Flat Surfaces, International Journal of Heat and Mass Transfer 13, 1873-1886.

[4] Hasanuzzaman, M., Saidur, R. Ali, M., Masjuki, H.H. 2007. Effects of Variables on Natural Convectve Heat Transfer trough V-Corrugated Vertical Plates, International Journal of Mechanical and Materials Engineering 2, 109-117.

[5] Qi, H.D., 2008. Fluid Flow and Heat Transfer Characteristics of Natural Convection in a Square Cavities due to Discrete Source-Sink Pairs, Interntaional Journal of Heat and Mass Transfer 51, 25-26.

[6] Nor Azwadi, C.S., Tanahashi, T., 2006. Simplified Thermal Lattice Boltzmann in Incomressible Limit, International Journal of Modern Physics B 20, 24372449.

[7] Laguerre, O., Amara, S.B., Flick, D., 2005. Experimental Study of Heat Transfer by Natural Convection in a Closed Cavity: Application in a Domestic Refrigerator, J. Food Engineering 70, 523537.

[8] Ravnik, J., Skerget, L., Zunic, Z., 2008. VelocityVorticity Formulation for 3D Natural Convection in an Inclined Enclosure by BEM, International Journal Heat and Mass Transfer 51, 4517-4527.

[9] Yasin, V., Hakan, F.O., Ahmet, K., Filiz, O., 2009. Natural Convection and Fluid Flow in Inclined Enclosure with a Corner Heater, Applied Thermal Engineering 29, 340-350.

[10] Kobus, C.J., 2005. Utilizing Disk Thermistors to Indirectly Measure Convective Heat Transfer Coefficients for Forced, Natural and Combined (Mixed) Convection, Experiments in Thermal and Fluid Science 29, 659-669.

[11] Laguerre, O., Remy, D., Flick, D., 2009. Airflow, Heat and Moisture Transfers by Natural Convection in a Refrigerating Cavity, J. Food Engineering 91, 197210.

[12] Nor Azwadi, C.S., Tanahashi, T., 2007. ThreeDimensional Thermal Lattice Boltzmann Simulation of Natural Convection in a Cubic Cavity, International Journal of Modern Physics B 21, 87-96.

[13] Lo, D.C., Young, D.L., Tsai, C.C., 2007. High Resolution of 2D Natural Convection in a Cavity by the DQ Method, J. Computers and Applied Mathematics 203, 219-236.

[14] Hasanuzzaman, M., Saidur, R and Masjuki, H.H., 2009. Effects of operating variables on heat transfer, energy losses and energy consumption of household refrigerator-freezer during the closed door operation, Energy 34(2), 196-198.

[15] Patrick, H.O., David, N., 1999. Introduction to Convective Heat Transfer Analysis. McGraw Hill.

[16] N.A.C. Sidik and M. R. A. Rahman, 2009. Cubic Interpolated Pseudo Particle (CIP)- Thermal BGK Lattice Boltzmann Numerical Scheme for solving Incompressible Thermal Fluid Flow Problem, Malaysian Journal of Mathematical Sciences, 3(2), 183-202.

[17] C.S.N. Azwadi and M.S.Idris, 2010. Finite Different and Lattice Boltzmann Modelling For Simulation of Natural Convection in A Square Cavity, International Journal of Mechanical and Materials Engineering (IJMME), Vol. 5, No.1, 80-86. 